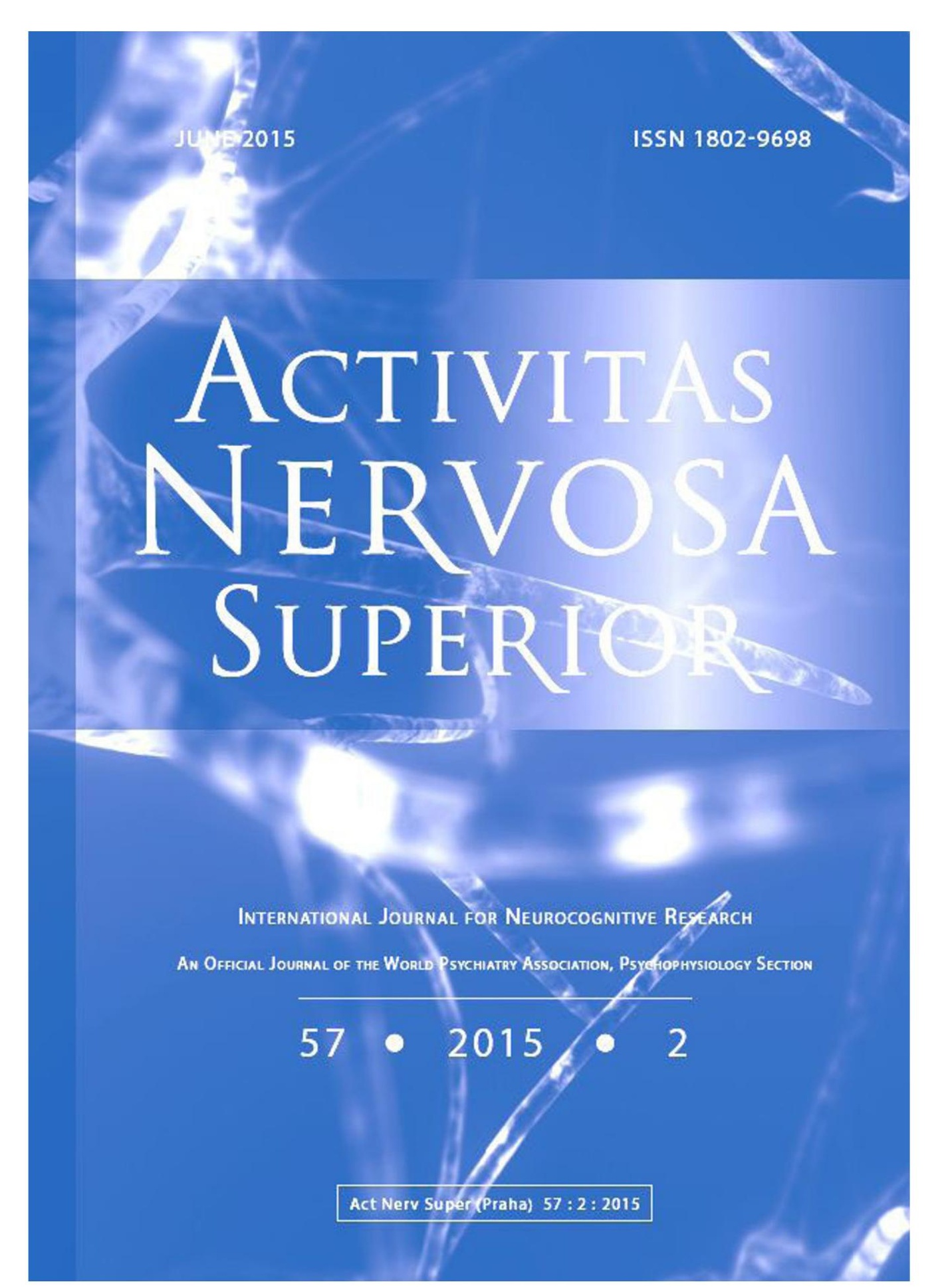

ANS: Journal for Neurocognitive Research Homepage:

www.activitas.org

\title{
CROSS SECTION CASE CONTROL STUdy For Clinical Correlates of COGNITIVE IMPAIRMENT IN PATIENTS WITH MIDLIFE EARLY HYPERTENSION: DIFFERENTIAL EFFECT OF ANTIHYPERTENSIVES
}

\author{
Hatem Elmassry ${ }^{1}$, Nahla Nagy² ${ }^{*}$ Iman Shorab ${ }^{2}$, Maissa Eid ${ }^{2}$ \\ ${ }^{1}$ Department of Neurology, Beni Suef University, Beni Suef, Egypt \\ 2Institute of Psychiatry, Ain Shams University, Cairo, Egypt
}

\begin{abstract}
High blood pressure (BP) is considered a risk factor for cerebrovascular disease, including stroke. Little is known about the importance of $\mathrm{BP}$ on the progression of microvascular disease of the brain, and cognitive impairment. In this study 85 patients (45 women and 40 men; age range 40-55) were assessed for blood pressure with the mean value of the 2 measurements of 2 visits that were calculated and analysed. Cognitive testing was performed using a combination of 4 well-validated standardized tests: the Mini Mental State Examination, Trail Making Test (TMT) A, (TMT) B, and a verbal fluency test. The control group of 60 normal volunteers matched for age, sex and education without changes in blood pressure were compared for cognitive changes. Result show significant difference between patients and controls in cognitive tests $(\mathrm{p}=0.000)$ and positive correlation between hypertension and cognitive impairment, that was more associated with elevated systolic blood pressure, older age, male sex and early onset hypertension. Positive correlation was also found with improved cognitive functions in hypertensive patients using diuretics, angiotensin II inhibitors and antihypertensive combination. The results indicate that midlife early high BP levels increase the risk for cognitive impairment and dementia. This association may be modified by antihypertensive medication.
\end{abstract}

Key words: Blood pressure; Cognitive impairment; Genetics; White matter lesions; Antihypertensives

\section{INTRODUCTION}

High blood pressure (BP) is considered a risk factor for cerebrovascular diseases, including stroke. Cognitive impairment is common among the elderly and can be an early symptom of dementia. Previous studies suggest that high BP levels increase the risk for late-life cognitive impairment and dementia (Launer et al., 1995; Skoog et al., 1996; Guo et al., 1999). Midlife high blood pressure has a strong adverse effect on cognitive function in persons with higher genetic susceptibility (Peila et al., 2001).

"Correspondence to: Nahla Nagy, e-mail: nahlanagy64@yahoo.com Received January 24, 2015; accepted June 10, 2015; Act Nerv Super 57(2), 71-80; ISSN-1802-9698 
An association of midlife high BP and cognitive impairment has been linked through multiple mechanisms. High BP can affect large and small vessels that penetrate the brain and in addition, other atherosclerotic conditions related to high BP, including stroke, chronic heart disease (CHD), and peripheral artery disease, alter cerebral flow autoregulation. As a consequence, transitory conditions of cerebral hypoxia/ischemia can occur, creating impaired cerebral perfusion and asymptomatic depression of oxygenation. Ischemia-related injuries can ultimately lead to clinical and subclinical brain damage, including lacunes and white matter abnormalities on MRI (Viitanen \& Guo, 1997; Stranggard et al., 1973; Faraci \& Heistad, 1990; Van Swieten et al., 1991).

Genetic factors APOE are hypothesized to play a central role in the response to neuronal injury by maintaining the integrity of the microtubules and redistributing lipids to regenerating neuronal axons (Handelmann et al., 1992). This neuroprotective function is highly allele specific: $A P O E \varepsilon 3$ seems to promote the repair process and APOE $\varepsilon 4$ to retard it both in vitro and in vivo (Horsburgh et al., 1999; Nathan et al., 1994; Bellosta et al., 1999; Teter et al., 1999; Buttini et al., 1999).

It is also possible that the effect of $A P O E$ on cognition is partially due to modifying the damage created by hypertension. Under this hypothesis, the impact of the hypertension would be much greater among $A P O E \& 4$ carriers due to their limited capacity to repair neuronal damage. APOE $\varepsilon 4$ is also associated with a lower concentration of plasma APOE protein and increased level of cholesterol and atherosclerosis, which can interact with hypertension and worsen atherosclerotic conditions (Boerwinkle et al., 1988; Davignon et al., 1988).

Inherent mechanisms in brain vessels keep cerebral blood flow constant despite variations in blood pressure (Van Beek et al., 2008). Once advanced cerebrovascular damage is present, these mechanisms cannot effectively regulate level of cerebral blood flow (Matsushita et al., 1994; Sierra et al., 2004). Therefore, low systemic blood pressure in the presence of cerebrovascular damage may lead to decline in cerebral blood flow, which renders brain tissue vulnerable to ischemic events (Kim et al., 2011).

Fluctuations of blood pressure (BP) over 24 hours and genetic predisposition can lead to vascular damage and white matter lesions (WMLs) that may contribute to cognitive deficits and the volume of periventricular confluent WMLs increases with increasing daytime BP (regression coefficient .47, 95\% confidence interval [CI], .13 to .71 vs .02, 95\% CI, -.32 to .36, $p$ $=.003$ for the difference between slopes). The volume of other WML subtypes (periventricular caps and rim, subcortical punctate, and subcortical confluent) was not associated with ambulatory BP. Participants carrying both CST3*B and CYP46*T alleles were overrepresented in the mild cognitive impairment group (MCI) with mild to severe WMLs (43\% vs 17\%, p.03). Thus BP and gene putative risk factors for cerebrovascular disease are differentially associated with WMLs in MCI patients of different WML severity. WMLs might develop for the convergence of innate with acquired factors (Galluzzi et al., 2008).

From the above studies it appears that the effects of increased blood pressure in the presence of genetic predisposition increase the liability of brain insults and hence cognitive impairment. Yet, these raises lots of questions as, when this hypertension presents itself clinically (onset of increased BP), to what extent can antihypertensives show modifying effect on coming complications and especially according to the scope of this paper on cognitive impairment. Do antihypertensives have differential effect in preventing cognitive decline, does this effect differ in some demographic variables as age and sex.

In this context, little is known about the importance of BP on the progression of microvascular disease of the brain, and cognitive impairment. This study aims to answer the above questions, to examine the onset of hypertension, the development of cognitive impairment, and the correlation with type of hypertension systolic and diastolic blood pressure, type of antihypertensive drugs, age and sex in comparison with matched normal control subjects. 


\section{METHODS}

\subsection{Participants}

In the cross-sectional design the subjects included in the study were divided into two groups, the patients and the control group. The patients included in the study were recruited from cardiology out and inpatient departments from Beny Sweif and Ain Shams University hospitals after approval of the study protocol from the concerned ethical committee in both medical departments.The control group was recruited from employees working in the hospital outpatient clinic. Data collection took place from January 2014 to October 2014.

\subsection{Procedures}

This work has been carried out in accordance with code of ethics of the Word Medical Association and written informed consent was obtained from all subjects participating in the study. The patients were examined and diagnosed by a cardiologist specialist who assessed blood pressure and referred patients to the neuropsychiatry department to assess medical history and cognitive functions. Inclusion criteria for patients were hypertension and comparable midlife age. Out of 120 (as calculated by Graphpad StatMate) 85 patients (45 women and 40 men) with clinical hypertension participated in the study. This group of patients also agreed to complete the cognitive tests. The control group was composed of 60 normal volunteers matched for age, sex, education without changes in blood pressure or significant medical or neurological disease.

\subsubsection{Blood pressure measure}

Blood pressure measures and clinical data were available from all participants. Systolic blood pressure (SBP) and diastolic blood pressure (DBP) were measured at baseline, in seating position at rest. The mean value of the 2 measurements of 2 visits was calculated and analysed. Detailed medical history including family history of hypertension, onset of hypertension and drug treatment was collected. Pharmacological treatment with agents affecting BP ( $\beta$-blockers, calcium antagonists, angiotensin-converting enzyme inhibitors and diuretics), was recorded in clinical history. Hypertension was the only vascular risk factor to avoid bias of other variables. Exclusion criteria included obesity (body mass index $>30$ $\left.\mathrm{kg} / \mathrm{m}^{2}\right)$, current smokers, those with abnormal laboratory tests for lipid profile, diabetes mellitus, liver and renal functions and significant medical or neurological disease (i.e. occurrence of clinically recognized stroke during the past 5 years according to the World Health Organization definition of stroke of rapidly developing clinical signs of focal disturbance of cerebral functioning lasting $>24$ hours to identify subjects with stroke events). The patients group was comparable with regard to sex, age, anthropometric characteristics and pharmacological treatment.

\subsubsection{Cognitive measures}

Cognitive testing was performed using a combination of 4 well-validated standardized tests: the Mini Mental State Examination, Trail Making Test (TMT A), (TMT B), and a verbal fluency test.

The mini-mental status examination (MMSE) test, is a brief 30-points questionnaire used to screen for cognitive impairment (Folstein et al., 1975). MMSE is commonly used to screen for Dementia. It samples various functions including arithmetic, memory, and orientation. A score greater than or equal to 25 points is effectively normal. Scores below 25 points can indicate severe (<9points) moderate (10-20 points), or mild (21-24 points) illness. Low to very low scores correlate closely with the presence of dementia. 
Verbal fluency test (VFT) (Borkowski et al., 1967), consisting of four items which grades the storage of semantic memory and retrieval. The VFT has been shown to be impaired in different dementias and frontal lobe dysfunctions. TrailMaking Test (TMT) (Borkowski et al., 1967), TMT part A consists of three items and evaluates the ability to solve problems under time pressure. It also requires visuoperceptual and psychomotor abilities. The TMT part B measures response inhibition and vulnerability to interference. The TMT (parts A and B) has been shown to be sensitive to impairment in cognitive function and inflexibility of response.

\subsection{Statistical analysis}

Collected data were analysed using statistical package for social sciences SPSS version 17. Qualitative variables were described in number and percentages. Quantitative variables were described as means and standard deviations. Pearson Chi Square test was used to detect presence or absence of significant association between two categorical variables. Independent sample t-test was used to compare means of different groups of patients. ANOVA tests were used for comparing quantitative data between more than two groups. $\mathrm{P}$ value was used to indicate the level of significance $(\mathrm{p}<0.05$ is considered significant, $\mathrm{p}<0.01$ is considered highly significant). Linear relationships were examined using Pearson's correlation coefficient.

\section{RESULTS}

At the beginning of the study 120 patients aged 40-55 years were examined for hypertension by specialist in the cardiology department and then they were referred to the neuropsychiatry department for cognitive test assessments. From this sample only 85 subjects agreed to complete the study procedures.

Their mean age was $43.9+6.2$ years, $M / F$ ratio $40 / 45$, their blood pressure means for systole 138.9+5.6 and diastole 90.2+2.9. 72 patients $(84.7 \%)$ showed positive family history for hypertension with mean onset of hypertension at $41.3+3.12$ years. The control group included 60 normal subjects, mean age (42-58), M/F 27/33 ratio and mean blood pressure for systole $(115.83+4.21)$ and diastole $(73.66+3.61)$. They were compared with the patient group for age $(p=0.3)$, sex $(p=1.3)$ and educational level $(p=1.2)$ with no statistical significance as shown in table 1.

Further results are in Table 2 that shows significant differences between patients and controls in cognitive tests MMSE and TMT $(p<0.05)$. There were positive correlations $(r>0.5)$ between hypertension and cognitive impairment that were more associated with systolic blood pressure and between cognitive impairment and increased age, male sex and early onset hypertension. Positive correlation with improved cognitive functions in hypertensive patients using diuretics, angiotensin II inhibitors and antihypertensive combination was found as shown in table 3.

We found positive correlations $r>0.5$ between cognitive impairment and increased age, male sex, early onset of hypertension, increased systolic blood pressure and antihypertensive drugs (Table 3).

\section{DISCUSSION}

This study focused on midlife hypertension and tried to answer some questions as early stated in our objectives. Hypertension runs in families with genetic background as $84.7 \%$ of our patients showed positive family history. It starts at an early onset with range of 33-46 years and mean+standard deviation $41.3+3.12$.The onset of hypertension starts earlier in males than females, yet it didn't reach levels of significance $(p=0.2)$. The mean levels for systolic and diastolic hypertension were significantly comparable between patients and controls as the 
only variable $(p=0.00)$ to avoid bias. We examined both groups for cognitive functions and found significant impairment in the patient group $(p<0.05)$.Linear regression analysis showed positive correlation with confidence interval $95 \%$ between cognitive impairment and increased age, male sex ,early onset of hypertension more for systolic blood pressure and improved cognitive functioning with Angiotensin II inhibitors ,Diuretic and Antihypertensive combination $(r>0.5)$.

Table 1. The above data shows significant differences between patients and controls between means of systolic and diastolic blood pressure $p=0.000$.

\begin{tabular}{|c|c|c|c|}
\hline Variable & Patient group & Control group & P ralue \\
\hline Number & 85 & $\infty 0$ & \\
\hline Age range & $40-55$ & $42-58$ & \\
\hline $\mathbf{M}+\mathbf{S D}$ & $43.9+62$ & $45+9.0$ & 0322 \\
\hline Sex & & & 13 \\
\hline M/ F ratio & $40 \cdot 45$ & $27: 33$ & \\
\hline $\mathrm{M} / \mathrm{F} 06$ & $47.05 \% \cdot 52.94 \%$ & $45 \% \cdot 55 \%$ & \\
\hline Educational lerel & & & 12 \\
\hline Illetrate & $27 \quad 31.7 \%$ & $18 \quad 30 \%$ & \\
\hline High school lerel & $38 \quad 44.70 \%$ & $28 \quad 46.6 \%$ & \\
\hline University lecel & $20 \quad 2352 \%$ & $14 \quad 23.3 \%$ & \\
\hline $\begin{array}{l}\text { Positive family history } \\
\text { of hy pertension } \\
96\end{array}$ & $\begin{array}{l}+ \text { ve } \therefore \text { - ve } \\
72 \cdot 13 \\
84.7 \% \cdot 15.2 \%\end{array}$ & - & - \\
\hline Onset of hypertension & & - & - \\
\hline Range in years & $33-46$ & & \\
\hline $\mathbf{M}+\mathbf{S D}$ & $41.3+3.12$ & & \\
\hline Systole blood pressure & $135-148$ & $120-130$ & 0.000 \\
\hline $\mathbf{M}+\mathbf{S D}$ & $138.9+5.6$ & $115.83+421$ & \\
\hline Diastole bbod pressure & $85-95$ & $70-80$ & 0.000 \\
\hline $\mathbf{M}+\mathbf{S D}$ & $90.2+29$ & $73.66+3.61$ & \\
\hline \multicolumn{4}{|l|}{$\begin{array}{l}\text { Antihypertensive drugs } \\
\text { Cardioselectire beta blocker } \\
\text { Ind eral, Tenormine }\end{array}$} \\
\hline & $8 \quad 9,4 \%$ & & \\
\hline \multicolumn{4}{|l|}{ Calcium channel } \\
\hline \multicolumn{4}{|l|}{ blockersIsopten,Ditia em } \\
\hline \multicolumn{4}{|l|}{ Angiotensin conrerting } \\
\hline \multicolumn{4}{|l|}{ enz rme inhibitor Capoten } \\
\hline & $23 \quad 27.05 \%$ & & \\
\hline \multicolumn{4}{|l|}{$\begin{array}{l}\text { Angiotensin II } \\
\text { inhibitors A tacand }\end{array}$} \\
\hline & $1720 \%$ & & \\
\hline \multicolumn{4}{|l|}{ Diuretic Lasix,Moduretic } \\
\hline & $34 \quad 40 \%$ & & \\
\hline Antihypertensice combination & $3237.64 \%$ & & \\
\hline $\begin{array}{l}\text { A tacand HCL Exforge, } \\
\text { Capoz ide }\end{array}$ & $42 \quad 49.41 \%$ & & \\
\hline
\end{tabular}

Table 2. Changes in cognitive functions in both patients and controls.

\begin{tabular}{llll}
\hline Neuropsychological Tests & Patients & Control & $\begin{array}{l}\text { p- } \\
\text { value }\end{array}$ \\
\hline MMSE & $19.8+2.3$ & $24.8+1.7$ & 0.0008 \\
TMT A & $50.6+4.2$ & $43.8+3.1$ & 0.0215 \\
TMT B & $150.8+3.9$ & $132.2+6.37$ & 0.0005 \\
VFT & $16.6+3.6$ & $20.4+3.64$ & 0.138 \\
\hline
\end{tabular}

Note. Significant difference between hypertensive patients and normal controls in cognitive functions $\mathrm{p}<0.05$. 
Table 3. Correlations between cognitive functions in hypertensive patients with different clinical variables.

\begin{tabular}{lcc}
\hline Age & Cognitive functions & R \\
\cline { 2 - 3 } \cline { 2 - 3 } Sex male & MMSE & -0.67 \\
TMT A & 0.53 \\
TMT B & 0.32 \\
Verbal fluencys & -0.30 \\
& MMSE & 0.54 \\
Onset blood pressure & TMT A & 0.51 \\
& TMT B & 0.42 \\
& Verbal fluency & 0.21 \\
& & \\
Systolic blood pressure & MMSE & 0.56 \\
& TMT A & -0.01 \\
& TMT B & -0.02 \\
& Verbal fluency & 0.03 \\
& & -0.62 \\
& MMSE & 0.57 \\
& TMT A & 0.37 \\
\hline
\end{tabular}

Recent studies have shown that increased diurnal blood pressure (BP) is closely associated with cognitive impairment via injury of the small cerebral arteries indicating that long-standing hypertension constitutes a risk of brain matter atrophy or white matter lesions (WMLs). In several clinical trials, BP-lowering with antihypertensive agents was suggested to reduce the risk of dementia or cognitive decline (Nagai et al., 2010).

Multiple variables correlated positively with cognitive impairment as male sex (odds ratio $[\mathrm{OR}]=1.59,95 \%$ confidence interval $[\mathrm{CI}] 1.43-1.77)$, black race $(\mathrm{OR}=2.09,95 \% \mathrm{CI} 1.88-2.35)$, less education (less than high school graduate vs college graduate, OR $=2.21,95 \%$ CI 1.88 2.60), older age (10-year increments, $\mathrm{OR}=2.11$, per 10-year increase in age, 95\% CI 2.05-2.18), and presence of left ventricular hypertrophy $(\mathrm{LVH}, \mathrm{OR}=1.29,95 \% \mathrm{CI} 1.06-1.58)$ and elevated systolic blood pressure was related to incident cognitive impairment (Unverzagt et al., 2011). These findings coincides with our results of positive correlation with confidence interval 95\% between cognitive impairment and increased age, male sex, early onset of hypertension more for systolic blood pressure.

However, the picture is not that simple as changes and effect of blood pressure varies between systole and diastole, across life span from midlife to old age and among both sexes.

Another study showed that increased diastolic blood pressure (DBP) levels were associated with impaired cognitive status after adjusting for demographic and environmental characteristics, risk factors, depressive symptoms, and antihypertensive medications. An increment of $10 \mathrm{~mm} \mathrm{Hg}$ in DBP was associated with a 7\% (95\% confidence interval [CI] 1\%$14 \%, p=0.0275$ ) higher odds of cognitive impairment (Tsivgoulis, 2009).

All longitudinal studies suggest that high midlife BP is a risk factor for later-life cognitive impairment and dementia, and that a low DBP and a very high SBP in older adults may be associated with subsequent development of dementia. Cerebral hypoperfusion secondary to 
severe atherosclerosis resulting from long-standing hypertension and low BP may be a major biological pathway linking both high BP in midlife and low BP in later life to cognitive decline and dementia (Jung \& Kim, 2013). Increased systolic BP, in 24 month follow-up study was associated with increased white matter hyperintensity volume in magnetic resonance imaging associated with cognitive impairment (White et al., 2011). A study performed in Uppsala, Sweden, reported that the diastolic BP (DBP) measured in midlife is related to global cognitive dysfunction at the age of 70 years (Kilander et al., 1998), while another study conducted in Kuopio and Joensuu, Finland, demonstrated that midlife hypertension and dyslipidemia increased the risk for Alzheimer's disease in later life (Elias et al., 1993).

Kuusisto et al. (1993) showed that age and education affect cognitive performance in hypertensive patients especially in women. Antihypertensives might succeed in controlling clinical blood pressure but to what extent can affect pathological changes in small blood vessels and preserve cognitive functions and do they have differential effect. In our study we found positive correlation with improved cognitive functions in hypertensive patients using diuretics, angiotensin II inhibitors and antihypertensive confidence interval 95\% ( $r>0.5)$.

Recent Japanese study examined the associations between classes of antihypertensive medication and the risk of cognitive impairment. $\beta$-blocker was consistently associated with a lower risk of cognitive impairment (incidence rate ratio [IRR] 0.69; 95\% confidence interval [CI] 0.50-0.94), as compared with men not taking any antihypertensive medications. The use of diuretics, calcium channel blockers, angiotensin-converting enzyme inhibitors, or vasodilators alone was not significantly associated with cognitive impairment. Results were similar excluding those with cardiovascular disease or $<1$ year of follow-up, and additionally adjusting for pulse pressure, heart rate, baseline and midlife systolic blood pressure, and midlife antihypertensive treatment (IRR 0.65; 95\% CI 0.45-0.94). The association between $\beta-$ blocker use and cognitive impairment was stronger among men with diabetes, men aged $>75$ years, and those with pulse pressure $\geq 70 \mathrm{~mm} \mathrm{Hg}$ (Gelber et al., 2013). Another study examined use of diuretics, angiotensin-1 receptor blockers (ARB), angiotensin-converting enzyme inhibitors (ACE-I), calcium channel blockers (CCB), or $\beta$-blockers (BB) was associated with a reduced risk of Alzheimer disease (AD) dementia in participants with normal cognition or mild cognitive impairment (MCI). The study followed up old patients at least 75 years of age with normal cognition or MCI over a median 6.1-year period. Diuretic use was reported by $15.6 \%$, ARB $6.1 \%$, ACE-I 15.1\%, CCB 14.8\%, and BB 20.5\%. From these 2,248 participants, $290(13 \%)$ developed AD dementia.

Hazard ratio for incident AD dementia among participants with normal cognition was 0.51 in diuretic (95\% confidence interval [CI] 0.31-0.82), 0.31 in ARB (95\% CI 0.14-0.68), 0.50 in ACE-I (95\% CI 0.29-0.83), 0.62 in CCB (95\% CI 0.35-1.09), and 0.58 in BB (95\% CI 0.36-0.93) users and was not significantly altered when mean systolic blood pressure was above $140 \mathrm{~mm}$ $\mathrm{Hg}$. In participants with $\mathrm{MCI}$, only diuretic use was associated with decreased risk (hazard ratio $=0.38,95 \%$ CI 0.20-0.73) (Yasar et al., 2013).

Factors that might interfere with the effect of antihypertensives can be seen as biopsychosocial. Genetic effect and its protective role on the integrity of blood vessel wall and neuronal regeneration, other medical diseases as diabetes and cardiac diseases, psychological aspect for hypertensive patients suffering from anxiety and depression especially those with type A personality and special races and eating habits might be protective or increase the risk of hyperlipidemia and atherosclerosis.

The relationship of Mediterranean diet (MeD), the incident cognitive impairment (ICI) and the interaction of race and vascular risk factors was studied. Adherence to a MeD (scored as 09) was computed from Food Questionaire. Cognitive status was evaluated at baseline and annually during a mean follow-up period of $4.0 \pm 1.5$ years using Six-item-Screener. ICI was identified in 1,248 (7\%) out of 17,478 individuals fulfilling the inclusion criteria. Higher adherence to MeD was associated with lower likelihood of ICI before (odds ratio [lsqb]OR[rsqb] 0.89; 95\% confidence interval [lsqb]CI[rsqb] 0.79-1.00) and after adjustment for potential confounders (OR 0.87; 95\% CI 0.76-1.00) including demographic characteristics, 
environmental factors, vascular risk factors, depressive symptoms, and self-reported health status. There was no interaction between race $(p=0.2928)$ and association of adherence to $\mathrm{MeD}$ with cognitive status. However, they identified a strong interaction of diabetes mellitus $(p=0.0134)$ on the relationship of adherence to MeD with ICI; high adherence to MeD was associated with a lower likelihood of ICI in nondiabetic participants (OR 0.81; 95\% CI 0.70$0.94 ; p=0.0066$ ) but not in diabetic individuals (OR 1.27; 95\% CI 0.95-1.71; $p=0.1063$ ) (Tsivgoulis et al., 2013).

In conclusion, early onset hypertension may be an important risk factor for late life cognitive impairment especially in patients with genetic predisposition. Thus, treatment of high blood pressure may be effective in prevention of cognitive dysfunction.

The association between essential hypertension and impaired cognitive function has previously been reported in some clinical studies. We had the chance to study the relation of changes in blood pressure and cognitive functioning, cross section in midlife patients with early onset hypertension .Our results cannot be generalized as our sample was a convenient one, and yet, they should be considered as preliminary results, with scope for future studies, with larger samples, and follow up studies. The results of which would be more informative. Further research is needed to identify the underlying mechanisms responsible for cognitive dysfunction associated with increased BP variability using functional imaging techniques. In addition, examination of more correlations between different variables, such as smoking, abnormal lipid profile, hyperinsulinemia, significant medical or neurological diseases.

\section{REFERENCES}

Bellosta, S., Nathan, B.P., Orth, M., Dong, L.M., Mahley, R.W., \& Pitas, R.E. (1995). Stable expression and secretion of apolipoproteins E3 and E4 in mouse neuroblastoma cells produces differential effects on neurite outgrowth. Journal of Biological Chemistry, 270, 27063-27071.

Boerwinkle, E., \& Utermann, G. (1988). Simultaneous effects of the apolipoprotein E polymorphism on apolipoprotein E, apolipoprotein B, and cholesterol metabolism. American Journal of Human Genetics, $42,104-112$.

Borkowski, J.G., Benton, A.L., \& Spreen, O. (1967). Word fluency and brain damage. Neuropsychologia, 5, 135-140.

Buttini, M., Orth, M., Bellosta, S., Akeefe, H., Pitas, R.E., Wyss-Coray, T., Mucke, L., \& Mahley, R.W. (1999). Expression of human apolipoprotein E3 or E4 in the brains of Apoe-/- mice: isoform-specific effects on neurodegeneration. Journal of Neuroscience, 19, 4867-4880.

Davignon, J., Gregg, R.E., \& Sing, C.F. (1988). Apolipoprotein E polymorphism and atherosclerosis. Arteriosclerosis, 8, 1-21.

Elias, M.F., Wolf, P.A., D'Agostino, R.B., Cobb, J., White, L.R. (1993). Untreated blood pressure level is inversely related to cognitive functioning: the Framingham Study. American Journal of Epidemiology, 138, 353-364.

Faraci, F.M., Heistad, D.D. (1990). Regulation of large cerebral arteries and cerebral microvascular pressure. Circulation Research, 66, 8-17.

Folstein, S.E., McHugh, P.R. (1975). Mini mental state .A practical method for grading the cognitive state of patients for the clinician. Journal of Psychiatric Research, 12, 189-198.

Galluzzi, S., Geroldi, C., Benussi, L., Ghidoni, R., Testa, C., Borsci, G., Bonetti, M., Manfellotto, D., Romanelli, G., Zulli, R., Binetti, G., \& Frisoni, G. (2008). Association of blood pressure and genetic background with white matter lesions in patients with mild cognitive impairment. Journals of Gerontology A, 63, 510 - 517.

Gelber, R., Ross, W., Petrovitch, H., Masaki, K., Launer, L., \& White, L. (2013). Antihypertensive medication use and risk of cognitiveimpairment: The Honolulu-Asia Aging Study. Neurology, 81, 888 $-895$.

Guo, Z., Fratiglioni, L., Zhu, L., Fasthom, J., Winblad, B., \& Viitanen, M. (1999). Occurrence and progression of dementia in a community poulation aged 75 years and older. Relationship of anitihypertensive medication use. Archives of Neurology, 56, 991-996. 
Handelmann, G.E., Boyles, J.K., Weisgraber, K.H., Mahley, R.W., \& Pitas, R.E. (1992). Effects of apolipoprotein E, beta-very low density lipoproteins, and cholesterol on the extension of neurites by rabbit dorsal root ganglion neurons in vitro. Journal of Lipid Research, 33, 1677-1688.

Horsburgh, K., Kelly, S., McCulloch, J., Higgins, G.A., Roses, A.D., Nicoll, J.A. (1999). Increased neuronal damage in apolipoprotein E-deficient mice following global ischaemia. Neuroreport, 10, 837-841.

Jung, H. \& Kim. K. (2013). Blood pressure variability and cognitive function in the elderly. Pulse, 1, 29-34.

Kilander, L., Nyman, H., Boberg, M., Hansson, L., \& Lithell, H. (1998). Hypertension is related to cognitive impairment: a 20-year follow-up of 999 men. Hypertension, 31, 780-786.

Kim, Y.S., Davis, S.C., Truijen, J., Stok, W.J., Secher, N.H., \& van Lieshout, J.J. (2011). Intensive blood pressure control affects cerebral blood flow in type 2 diabetes mellitus patients. Hypertension, 57, 738745.

Kuusisto, J., Koivisto, K., Mykkänen, L., Helkala, E. L., Vanhanen, M., Hänninen, T., Pyörälä, K., Riekkinen, P. \& Laakso, M. (1993). Essential hypertension and cognitive function. The role of hyperinsulinemia. Hypertension, 22, 771-779.

Launer, L.J., Masaki, K., Petrovich, H., Foley, D., \& Havlik, R.J. (1995). The association between midlife blood pressure and late-life cognitive function. JAMA, 274, 1846-1851.

Matsushita, K., Kuriyama, Y., Nagatsuka, K., Nakamura, M., Sawada, T., \& Omae, T. (1994). Periventricular white matter lucency and cerebral blood flow autoregulation in hypertensive patients. Hypertension, 23, 565-568.

Nagai, M., Hoshide, S., \& Kario, K. (2010). Hypertension and Dementia. American Journal of Hypertension, 23, $116-124$.

Nathan, B.P., Bellosta, S., Sanan, D.A, Weisgraber, K.H., Mahley, R.W., \& Pitas, R.E. (1994). Differential effects of apolipoproteins E3 and E4 on neuronal growth in vitro. Science, 264, 850-852.

Peila, R., White, L.R., Petrovich, H., Masaki, K., Ross, G. W., Havlik, R.J., Launer, L.J. (2001). Joint effect of the APOE gene and midlife systolic blood pressure on late-life cognitive impairment: the Honolulu-Asia aging study. Stroke, 32, 2882 - 2889.

Reitan, R.M. (1958). Validity of the Trail Making Test as an indicator of organic brain damage. Perceptual and Motor Skills, 8, 271-276.

Sierra, C., de la Sierra, A., Chamorro, A., Larrousse, M., Domènech, M., \& Coca, A. (2004). Cerebral hemodynamics and silent cerebral white matter lesions in middle-aged essential hypertensive patients. Blood Pressure, 13, 304-309.

Skoog, I., Lernfelt, B., Landahl, S., Palmertz, B., Andreasson, L.A., Nilsson, L., Persson, G., Oden, A.,\& Svanborg, A. (1996). 15-year longitudinal study of blood pressure and dementia. Lancet, 347, 11411145.

Stranggard, S., Oleson, J., Skluhoj, E., \& Lassen, N.A. (1973). Autoregulation of brain circulation in severe arterial hypertension. BMJ, 1, 507-510.

Teter, B., Xu, P.T., Gilbert, J.R., Roses, A.D., Galasko, D., \& Cole, G.M. (1999). Human apolipoprotein E isoform-specific differences in neuronal sprouting in organotypic hippocampal culture. Journal of Neurochemistry, 73, 2613-2616.

Tsivgoulis, G., Judd, S., Letter, A.J., Alexandrov, A.V., Howard, G., Nahab, F., Unverzagt, F.W., Moy, C., Howard, V.J., Kissela, B., \& Wadley, V.G. (2013). Adherence to a Mediterranean diet and risk of incident cognitive impairment. Neurology, 80, 1684 - 1692.

Tsivgoulis. G., Alexandrov, A.V., Wadley, V.G., Unverzagt, F.W., Go, R.C., Moy, C.S., Kissela, B., \& Howard, G. (2009). Association of higher diastolic blood pressure levels with cognitive impairment. Neurology, 73, 589 - 595.

Unverzagt, F.W., McClure, L.A., Wadley, V.G., Jenny, N.S., Go, R.C., Cushman, M., Kissela, B.M., Kelley, B.J., Kennedy, R., Moy, C.S., Howard, V., \& Howard, G. (2011). Vascular risk factors and cognitiveimpairment in a stroke-free cohort. Neurology, 77, 1729 - 1736.

Van Beek, A.H., Claassen, J.A., Rikkert, M.G., \& Jansen, R.W. (2008). Cerebral autoregulation: an overview of current concepts and methodology with special focus on the elderly. Journal of Cerebral Blood Flow and Metabolism, 28, 1071-1085.

Van Swieten, J.C., Geyskes, O.G., Derix, M.A., Peeck, B.M., Ramos, L.M., van Latum, J.C., \& van Gijn, J. (1991). Hypertension in the elderly is associated with white matter lesions and cognitive decline. Annals of Neurology, 30, 825-830.

Viitanen, M., \& Guo, Z. (1997). Are cognitive function and blood pressure related? Drugs \& Aging, 3, 165169.

White, W.B., Wolfson, L., Wakefield, D.B., Hall, C.B., Campbell, P., Moscufo, N., Schmidt, J., Kaplan, R.F., Pearlson, G., \& Guttmann, C.R. (2011). Average daily blood pressure, not office blood pressure, 
is associated with progression of cerebrovascular disease and cognitive decline in older people. Circulation, 124, 2312- 2319.

Yasar, S., Xia, J., Yao, W., Furberg, C.D., Xue, Q.L., Mercado, C.I., Fitzpatrick, A.L., Fried, L.P., Kawas, C.H., Sink, K.M, Williamson, J.D., DeKosky, S.T., Carlson, M.C., Ginkgo Evaluation of Memory (GEM) Study Investigators. (2013). Antihypertensive drugs decrease risk of Alzheimer disease: Ginkgo Evaluation of Memory Study. Neurology, 81, 896 - 903. 